\title{
Comparison of Contrast Sensitivity in Different Types of Ocular Deviation for Different Amount of Deviation
}

\author{
Partha Haradhan Chowdhury ${ }^{1 *}$ and Brinda Haren Shah ${ }^{2}$ \\ ${ }^{1}$ M.Optom, Associate Professor, Principal, Department of Optometry, Shree Satchandi \\ Jankalyan Samiti Netra Prasikshan Sansthan, Pauri, Affiliated to Uttarakhand State \\ Medical Faculty, Dehradun, India \\ 2M.Optom, Guest Lecturer, Department of Optometry, Shree Satchandi Jankalyan \\ Samiti Netra Prasikshan Sansthan, Pauri, Affiliated to Uttarakhand, State Medical \\ Faculty, Dehradun, India
}

*Corresponding author: Partha Haradhan Chowdhury, M. Optom, Associate Professor, Principal, Department of Optometry, Shree Satchandi Jankalyan Samiti Netra Prasikshan Sansthan, Pauri, Affiliated to Uttarakhand State Medical Faculty, Dehradun, India, Email: optometrypublish@gmail.com

\section{Abstract}

Purpose: Aim of the present study is to compare contrast sensitivity in different types of ocular deviation for different amount of deviation.

Methods: A pilot, cross sectional, observational study was performed at tertiary eye care centers. Subjects with Ocular deviation between 10 to 40 prism diopters, Corrected distance Visual Acuity should be greater than 6/18 and Age should be between 10 to 40 years of age were included in the study. Contrast sensitivity was assessed with Pelli Robson Chart.

Results: 30 subjects were included in the study. Out of that, 16 subjects were in the age group of 11-20 years, 12 subjects were in the age group of 21-30 years and 2 subjects were in the age group of 31-40 years. $60 \%$ subjects were Female and $40 \%$ subjects were Male. The mean contrast sensitivity was considered in each type and amount of deviation. It shows that maximum contrast sensitivity is deteriorated in Esotropia and in the ocular deviation of 31-40 prism diopters.

Conclusions: This concludes that with increase in ocular deviation, there is a deterioration of Contrast Sensitivity. Maximum Contrast Sensitivity is deteriorated in Esotropia which is followed by Exotropia, Alternate Esotropia and Alternate Exotropia.

Keywords: Contrast Sensitivity; Type of Deviation; Amount of Deviation

\section{Introduction}

Ocular deviations have a major role in Binocular Single Vision. However, it has a major role in Contrast Sensitivity also. In case of normal ocular deviation, the images of an object are placed on the foveal area. In the fovea, the cone density is highest. But in case of ocular deviation, the images are shifted according to the deviation. In case of 
Eso deviation, the image is shifted to the nasal para fovea and vice versa in Exo deviation. But, due to image shift it may create deterioration of contrast sensitivity. It occurs due to anatomical cone cell number variation. In the foveal region cone cell density is highest compared to para foveal region. In case of Eso deviation, there are more chances of deterioration as compared to intermittent esotropia, exotropia and intermittent exotropia. Because intermittent timing is very less compare to other ocular deviation. Hence, ocular deviation is a very important factor for Contrast Sensitivity [1,2].

\section{Methodology}

Pilot, Cross Sectional and observational study was performed at tertiary eye care centers. Inclusion criteria includes Subjects with Ocular deviation between 10 to 40 prism diopters, Corrected distance Visual Acuity should be greater than 6/18 and Age should be between 10 to 40 years of age. Individuals with any other systemic disease(specially which can affect study), Individuals with any other Ocular Pathology, with any active ocular infection, any ocular anomalies like Corneal Scar etc ,ocular deviation if less than 10 degree and Significant amount of amblyopic patient were excluded from the study. Full refractive correction along with detailed fundus evaluation was performed in each and every patient. Contrast sensitivity was assessed with Pelli Robson Chart. Data analysis was done using SPSS software version $20[3,4]$.

\section{Results}

30 subjects were included in the study. Age wise distribution is shown in Figure 1. 16, 12 and 2 subjects were in the age group of 11-20 years, 12 in 21-30 years and 2 subjects were in the age group of 31-40 years. Figure 2 shows gender wise distribution of the subjects. $60 \%$ subjects were Female and $40 \%$ subjects were Male. Figure 3 shows comparison of mean Contrast Sensitivity with each ocular deviation in every amount of ocular deviation.

Blue colour indicates amount of ocular deviation of 1120 prism diopters. Red colour indicates amount of deviation of 21-30 prism diopters. Green colour indicates amount of deviation of 31-40 prism diopters.

In the ocular deviation of ESOTROPIA, mean Contrast Sensitivity for 11-20, 21-30 and 31-40 prism diopters are $1.65,1.42,1.42$ respectively which shows that maximum

Partha Haradhan Chowdhury and Brinda Haren Shah. Comparison of Contrast Sensitivity in Different Types of Ocular Deviation for Different Amount of Deviation. J Ophthalmol 2018, 3(S2): 000S2-021. deterioration of Contrast Sensitivity is in 31-40 prism diopters of Esotropia.

In the ocular deviation of Exotropia, mean Contrast Sensitivity for 11-20, 21-30 and 31-40 prism diopters are $1.72,1.6,1.5$ respectively which shows that maximum deterioration of Contrast Sensitivity is in 31-40 prism diopters of Exotropia.

In the ocular deviation of Alternate Esotropia, mean Contrast Sensitivity for 11-20, 21-30 and 31-40 prism diopters are $1.85,1.8,1.65$ respectively which shows that maximum deterioration of Contrast Sensitivity is in 31-40 prism diopters of Alternate Esotropia.

In the ocular deviation of Alternate Exotropia, mean Contrast Sensitivity for 11-20, 21-30 and 31-40 prism diopters are 1.95, 1.87, 1.8 respectively which shows that maximum deterioration of Contrast Sensitivity is in 31-40 prism diopters of Alternate Exotropia.

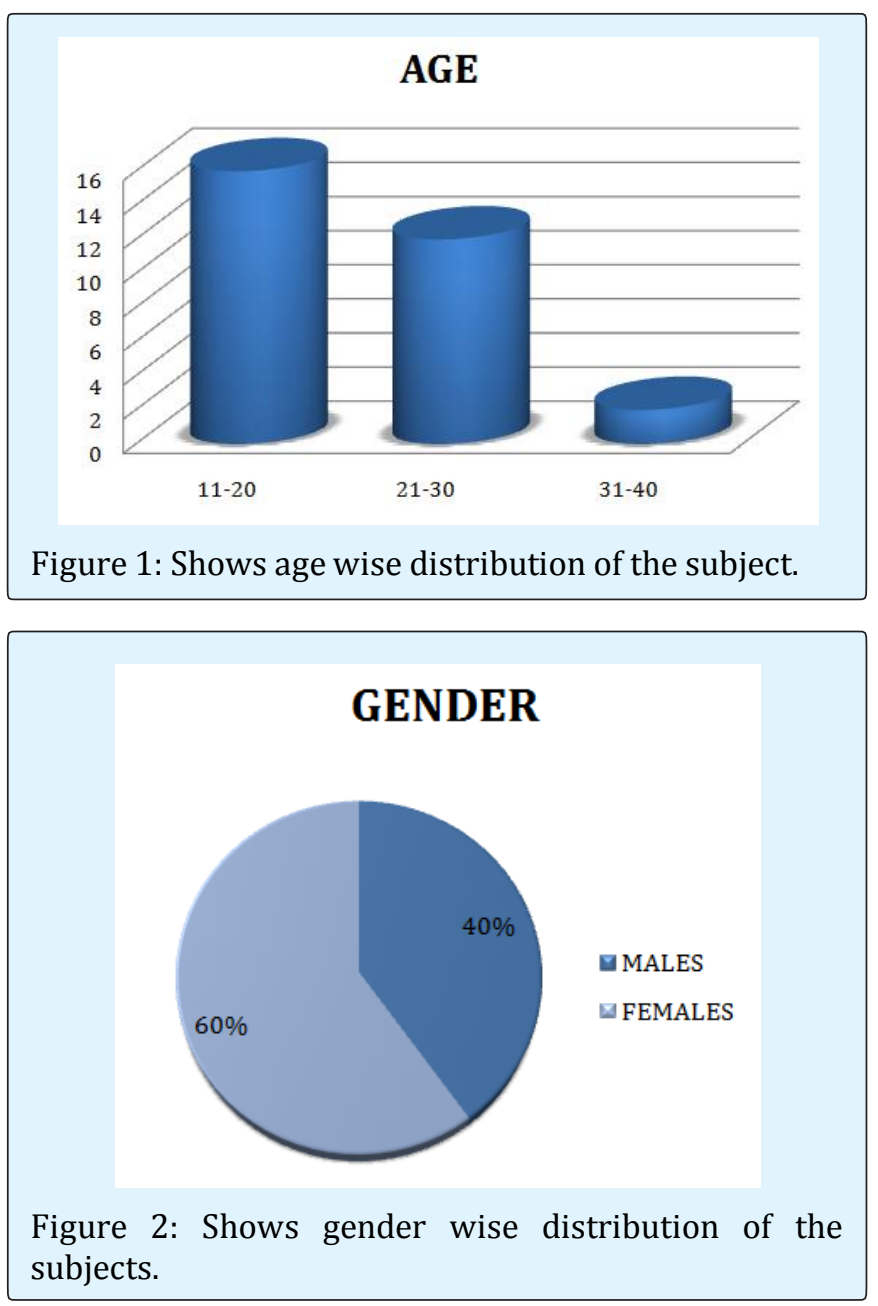

Copyright $($ Partha Haradhan Chowdhury and Brinda Haren Shah. 


\section{CONTRAST SENSITIVITY}

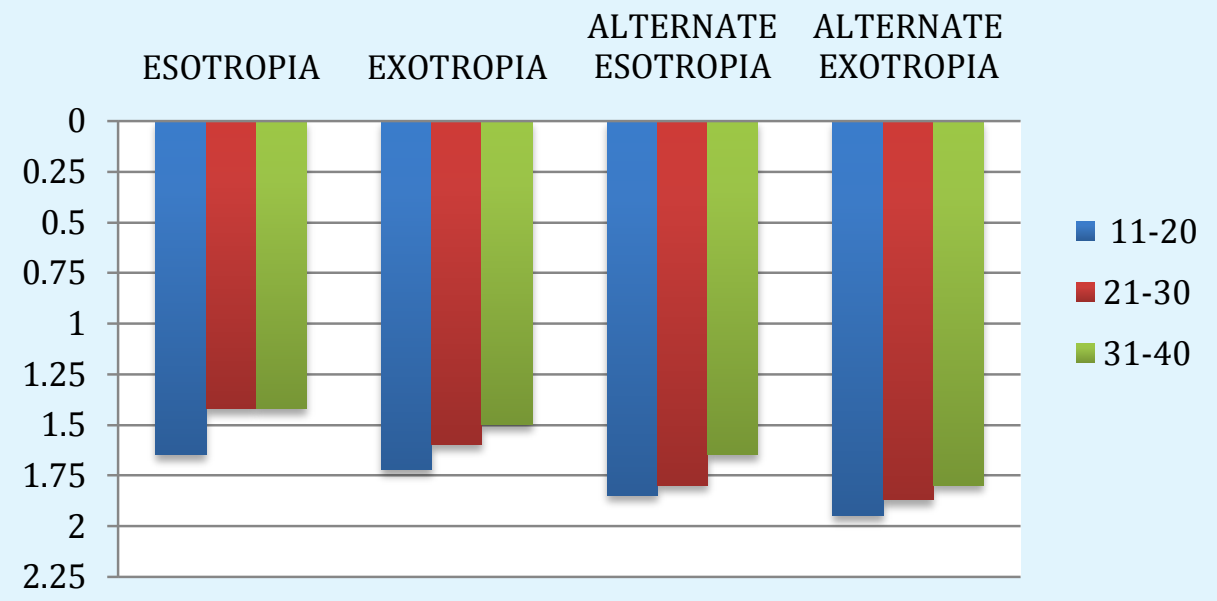

Figure 3: Shows comparison of mean Contrast Sensitivity with each ocular deviation in every amount of ocular deviation.

\begin{tabular}{|c|c|c|c|}
\hline Ocular Deviation & $\mathbf{1 1 - 2 0}$ & $\mathbf{2 1 - 3 0}$ & $\mathbf{3 1 - 4 0}$ \\
\hline Esotropia & 1.65 & 1.42 & 1.42 \\
\hline Exotropia & 1.72 & 1.6 & 1.5 \\
\hline Alternate Esotropia & 1.85 & 1.8 & 1.65 \\
\hline Alternate Exotropia & 1.95 & 1.87 & 1.8 \\
\hline
\end{tabular}

Table 1: Shows the mean values of contrast sensitivity with each ocular deviation in every amount of ocular deviation.

\section{Discussion}

According to the study, it can be concluded that in case of ocular deviation, Contrast Sensitivity is deteriorated. It mainly occurs due to anatomical consideration of the cone cell in the macular region. In the foveal area, cone density is highest compared to para foveal region. In cases of ocular deviation, images are shifted to para foveal region and that is the reason for deterioration of contrast sensitivity. As per this study, deterioration occurs more in Esotropia compared to Intermittent Esotropia, Exotropia and Intermittent Exotropia. So, ocular deviation is the concerned factor for contrast sensitivity [5-7].

\section{Conclusions}

This concludes that with increase in ocular deviation, there is a deterioration of Contrast Sensitivity. Maximum

Partha Haradhan Chowdhury and Brinda Haren Shah. Comparison of Contrast Sensitivity in Different Types of Ocular Deviation for Different Amount of Deviation. J Ophthalmol 2018, 3(S2): 000S2-021.
Contrast Sensitivity is deteriorated in Esotropia which is followed by Exotropia, Alternate Esotropia and Alternate Exotrop.

\section{References}

1. Handbook of Pediatric Strabismus and Amblvobia (2006) Kenneth W. Wright, ,Peter H. Spiegel Lisa ,Thompson $1^{\text {st }}$ (Edn.).

2. Hui Zhu, Jia-Jia Yu, Rong-Bin Yu, Hui Ding, Jing Bai, et al. (2015) Association between Childhood Strabismus and Refractive Error in Chinese Preschool Children. Plos One 10(6): e0130914.

3. Zhale Rajavi, Sabbaghi H, Baghini AS, Yaseri M, Sheibani K, et al. (2015) Prevalence of Colour Vision Deficiency and its Correlation with Amblyopia and Refractive Errors among Primary School Children. J Ophthalmic Vis Res 10(2): 130-138.

4. Anika K. Tandon, Federico G Velez, Sherwin J Isenberg, Joseph L Demer, Stacy L Pineles et al. (2014) Binocular Inhibition in Strabismic Patients is Associated with Diminished Quality of Life. Journal of American Association for Pediatric Ophthalmology and Strabismus 18(5): 423-426. 


\section{Open Access Journal of Ophthalmology}

5. XC Ye, Pegado V, Patel MS, Wasserman WW (2014) Strabismus genetics across a spectrum of eye misalignment disorders. Clin Genet 86(2): 103-111.

6. Kocak-Altintas AG (2000) Colour vision and Colour Vision deficiency in Amblyopia. European Journal of Ophthalmology 10(1): 77-81.
7. Freeman AW (1996) Components of Colour vision Loss in Strabismus. Journal of vision research 36(5): 765-774.

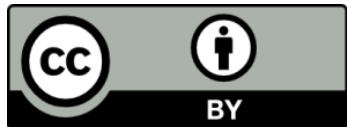

Partha Haradhan Chowdhury and Brinda Haren Shah. Comparison of Contrast Sensitivity in Different Types of Ocular Deviation for Different Amount of Deviation. J Ophthalmol 2018, 3(S2): 000S2-021.
Copyright@ Partha Haradhan Chowdhury and Brinda Haren Shah. 